\title{
Control de presión, modelado matemático y sintonización de controladores por el método de Ziegler-Nichols
}

\section{Pressure control, mathematical modeling and controller tuning by the Ziegler- Nichols method}

\author{
MARTÍNEZ-MARÍN, Francisco Alejandro†*, GARCIABADA-SILVA, Gabriel y HERNÁNDEZ- \\ CERVANTES, Aldo Aarón
}

Instituto Tecnológico de Jalisco José Mario Molina Pasquel y Henriquez, México.

ID $1^{\mathrm{er}}$ Autor: Francisco Alejandro, Martínez-Marín / ORC ID: 0000-0002-3713-5182

ID $1^{\mathrm{er}}$ Coautor: Gabriel, Garciabada-Silva / ORC ID: 0000-0002-7083-871X

ID $2^{\text {do }}$ Coautor: Aldo Aarón, Hernández-Cervantes / ORC ID: 0000-0001-8060-5509

DOI: $10.35429 /$ JIE.2020.13.4.8.12

Recibido Julio 15, 2020; Aceptado Diciembre 30, 2020

\section{Resumen}

La obtención de los parámetros de un controlador PID se realiza a través de varios pasos, el modelado matemático es el primero de ellos, donde se verifica que el número de incógnitas sea igual al número de ecuaciones. Haciendo uso de las transformadas de Laplace, se pasa del domino del tiempo al dominio de Laplace. Por el método de Ziegler-Nichols, y sustituyendo iw por s en la ecuación característica del proceso se pasa al dominio de la frecuencia y se establece la ganancia última y con la cual es posible deducir los valores de un controlador proporcional, derivativo e integral. La ganancia última obtenida de una manera analítica se puede comprobar por medio de las gráficas del lugar de las raíces, las cuales se pueden generar fácilmente por medio de programas en Matlab, pero esto queda fuera del alcance de este artículo.

Controladores, Ecuación característica, ZieglerNichols, Ganancia última, Factor de amortiguamiento

\begin{abstract}
Obtaining the parameters of a PID controller is done through several steps, mathematical modeling is the first of them, where it is verified that the number of unknowns is equal to the number of equations. Using the Laplace transforms, one passes from the time domain to the Laplace domain. By the Ziegler-Nichols method, and substituting iw for $\mathrm{s}$ in the characteristic equation of the process, the frequency domain is passed and the ultimate gain is established and with which it is possible to deduce the values of a proportional, derivative and integral controller. The final gain obtained in an analytical way can be verified by means of the graphs of the place of the roots, which can be easily generated by means of programs in Matlab, but this is beyond the scope of this article.
\end{abstract}

Drivers, Characteristic equation, Ziegler-Nichols, Ultimate gain, Damping factor

Citación: MARTÍNEZ-MARÍN, Francisco Alejandro, GARCIABADA-SILVA, Gabriel y HERNÁNDEZ-CERVANTES, Aldo Aarón. Control de presión, modelado matemático y sintonización de controladores por el método de Ziegler-Nichols. Revista de Ingeniería Industrial. 2020. 4-13:8-12.

\footnotetext{
*Correspondencia al Autor (Correo Electrónico: francisco.martinez@ vallarta.tecmm.edu.mx)

$\dagger$ Investigador contribuyendo como primer autor.
} 


\section{Introducción}

\section{Modelo para control de presión}

El módulo de procesos RT590 esta habilitado para realizar prácticas de control de presión haciendo uso del tanque \# 2, el aire a una presión máxima de 6 bars se introduce al sistema a través del la válvula PCV1, está válvula se opera en un inicio de una manera manual con el tablero de control previamente energizado, con el fin de obtener una presión de inicio en el tanque \#2, la presión sugerida es de 4 bars. Las válvulas manuales abiertas deben de ser las HV11 y HV17, se debe de tener especial cuidado en mantener la valvula de flujo FCV2 cerrada como también la bomba de agua apagada.El controlador PICA es el designado para realizar pruebas de presión por lo que se debe de poner en automático, mientras que el resto de los controladores de la consola permanecen en manual. Una vez realizados los preparativos anteriores se introducen los valores al controlador.La figura 3 indica la posición de las valvulas manuales HV11 y HV17y FCV2.

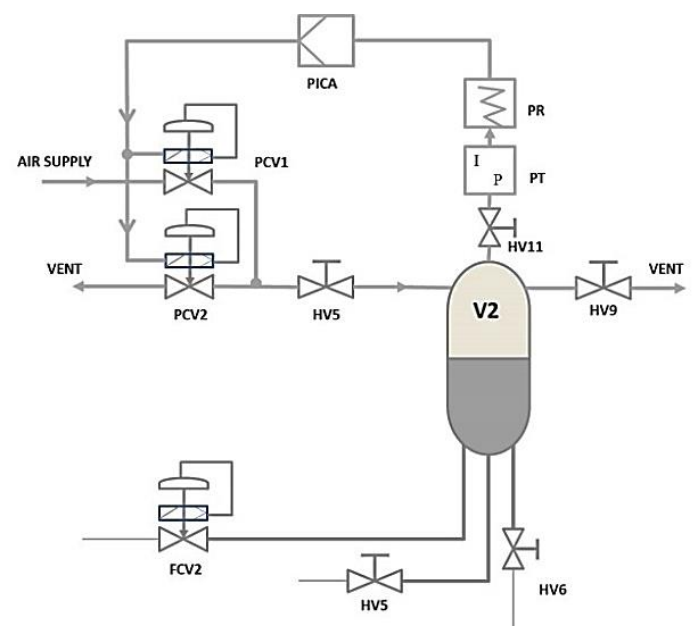

Figura 1 Esquema de procesos Control de presión

\section{Materiales y métodos}

\section{Consideraciones}

Temperatura constante de $25^{\circ} \mathrm{C}$ (Proceso isotérmico), el volumen se considera constante, la presión de suministro de aire es constante, tiempo en segundos.

\begin{tabular}{|l|l|r|l|}
\hline \multicolumn{5}{|c|}{ Variables } & Unidades & \multicolumn{2}{|c|}{$\begin{array}{c}\text { Condiciones } \\
\text { iniciales en } \\
\text { estado estable. }\end{array}$} \\
\hline$w_{1}$ & $\mathrm{~g} / \mathrm{s}$ & 1.0701 & $\begin{array}{l}\text { Variable de } \\
\text { entrada. }\end{array}$ \\
\hline$w_{2}$ & $\mathrm{~g} / \mathrm{s}$ & 1.0701 & $\begin{array}{l}\text { Variable de } \\
\text { entrada. }\end{array}$ \\
\hline $\mathrm{m}_{1}$ & $\mathrm{~g}$ & 72.0673 & $\begin{array}{l}\text { Variable de } \\
\text { estado. }\end{array}$ \\
\hline $\mathrm{p}_{2}$ & $\mathrm{bars}$ & 2.200 & $\begin{array}{l}\text { Variable } \\
\text { controlada. }\end{array}$ \\
\hline apcv1 & adimensional & 0.2847 & $\begin{array}{l}\text { Variable } \\
\text { manipulada. }\end{array}$ \\
\hline apcv2 & adimensional & $1-0.2847$ & $\begin{array}{l}\text { Variable } \\
\text { manipulada. }\end{array}$ \\
\hline
\end{tabular}

Tabla 1

Fuente: Elaboración propia

Las ecuaciones se resuelven en el orden que se indica.

apcv2=1-apcv1

Ley general de los gases.

$P_{2}=\left(\mathrm{m}_{1} / \mathrm{pma}\right) R T / \mathrm{v}_{2}$

La ecuación de la válvula de entrada está dada por:

$w_{1}=\operatorname{apcv} 1 * \operatorname{cvp} 1 \sqrt{\left(P_{1}^{2}-P_{2}^{2}\right) / 2}$

La ecuación de la válvula de salida está dada por la válvula PCV2 que se aprecia en el diagrama:

$w_{2}=\operatorname{apcv} 2 * \operatorname{cvp} 2 \sqrt{\left(P_{2}{ }^{2}-P_{a t m}{ }^{2}\right) / 2}$

Flujo subsónico

$w_{2}=0.85 * \operatorname{apcv} 2 * \operatorname{cvp} 2 * P_{2}$

Flujo sónico

Ecuación de continuidad. (Ecuación de estado).

$\frac{\mathrm{dm}_{1}}{\mathrm{dt}}=\mathrm{w} 1-\mathrm{w} 2$

Salida del controlador.

apcv $1=0.5+\mathrm{kp} * \mathrm{e}+\mathrm{ki} * \mathrm{ei}+\mathrm{kd} * \mathrm{de}$ 
Parámetros:

R, T, pma, v2, cvp2, Patm,

Variables de entrada: $\mathrm{p}_{1}$

Variables de estado: $\mathrm{p}_{2}$

Existen seis ecuaciones, 6 incógnitas, (w1, w2, m1, p2, apcv1, apcv2) cero grados de libertad.

Se descarta el flujo subsónico debido a que el flujo no está en función de la presión fluido- abajo (ecuación 4).

\section{Sintonización de controladores}

Control de Presión.

Función de transferencia y ecuación característica en lazo cerrado.

En 5 se sustituye apcv2 por 1 sustituyendo después 3 y 5 en 6 , esta última es la ecuación de continuidad, se genera una ecuación no lineal por lo que se recurre la linealización haciendo uso de la serie de Taylor.

$\frac{29 * \mathrm{~V}_{2}}{\mathrm{RT}} \frac{\mathrm{P}_{2}}{\mathrm{dt}}=\operatorname{apcv} 1 * \operatorname{cvp} 1 \sqrt{\left(\mathrm{P}_{1}{ }^{2}-\mathrm{P}_{2}{ }^{2}\right) / 2}-0.85 *(1-\operatorname{apcv} 1) * \operatorname{cvp} 2 * \mathrm{P}_{2}$

$\frac{29 * \mathrm{v}_{2}}{\mathrm{RT}} \frac{\mathrm{P}_{2}}{\mathrm{dt}}=\operatorname{apcv1} 1 * \operatorname{cvp} 1 \sqrt{\left(\mathrm{P}_{1}{ }^{2}-\mathrm{P}_{2}{ }^{2}\right) / 2}-0.85 * \operatorname{cvp} 2 * \mathrm{P}_{2}+0.85 * \operatorname{apcv} 1 * \operatorname{cvp} 2 * \mathrm{P}_{2}$

Linealizando, donde $\mathrm{V}$ es el volumen de casi 281 , la temperatura es de $25^{\circ} \mathrm{C}$, la presión a controlar es de 2,2 bars, la apertura de la válvula 1(apvc1) es de 0.2847, las constantes de las válvulas cvp1 y cvp2 son 0.8 para ambas, $\mathrm{R}$ constante universal de los gases es de 0.08314 .

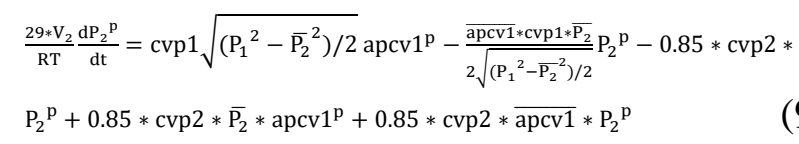

Transformando a Laplace.

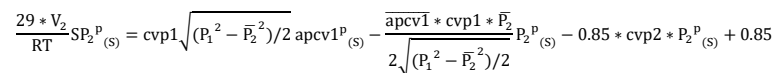

$$
\begin{aligned}
& * \operatorname{cvp} 2 * \overline{\mathrm{P}_{2}} * \operatorname{apcv1}^{\mathrm{p}}{ }_{(\mathrm{s})}+0.85 * \operatorname{cvp} 2 * \overline{\operatorname{apcv} 1} * \mathrm{P}_{2}^{{ }^{\mathrm{p}}}{ }_{(\mathrm{s})} \\
& \frac{\mathrm{P}_{2}{ }^{\mathrm{p}}(\mathrm{S})}{\operatorname{apcv}^{\mathrm{p}}{ }_{(\mathrm{S})}}=\frac{\operatorname{cvp} 1 \sqrt{\left(\mathrm{P}_{1}{ }^{2}-{\overline{\mathrm{P}_{2}}}^{2}\right) / 2}+0.85 * \operatorname{cvp} 2 * \overline{\mathrm{P}_{2}}}{\frac{29 * \mathrm{~V}_{2}}{\mathrm{RT}} \mathrm{S}+\frac{\overline{\mathrm{apcv1}} * \mathrm{cvp} 1 * \overline{\mathrm{P}_{2}}}{2 \sqrt{\left(\mathrm{P}_{1}{ }^{2}-\overline{\mathrm{P}}_{2}{ }^{2}\right) / 2}}+0.85 * \operatorname{cvp} 2-0.85 * \operatorname{cvp} 2 * \overline{\mathrm{apcv} 1}}
\end{aligned}
$$

Sustituyendo.

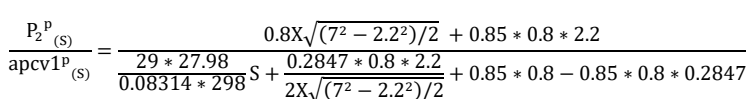

Función de transferencia en lazo abierto.

$$
\frac{\mathrm{P}_{2}{ }_{(\mathrm{S})}^{\mathrm{p}}}{\operatorname{apcv1}_{(\mathrm{S})}^{\mathrm{p}}}=\frac{5.2551}{32.75 \mathrm{~S}+0.5398}
$$

Considerando un retardo de $3 \mathrm{~s}$ por las dinámicas no consideradas, calculamos la aproximación de pade de primer orden.

$G_{\text {pade }}=\frac{-S+0.6667}{S+0.6667}$

Función de transferencia y ecuación característica en lazo cerrado.

Considerando:

$$
\begin{aligned}
G_{M} & =G_{M(s)} G_{\text {pade }} \\
\mathrm{G}_{\mathrm{M}} & =\left(\frac{5.2551}{32.75 S+0.5398}\right)\left(\frac{-S+0.6667}{S+0.6667}\right) \\
\mathrm{G}_{\mathrm{M}} & =\frac{-5.2551 S+3.5035}{32.75 \mathrm{~S}^{2}+22.373 \mathrm{~S}+0.35988}
\end{aligned}
$$

$\mathrm{B}_{(\mathrm{s})}$ Función de transferencia del controlador.

La ecuación característica en lazo cerrado es:

Considerando:

$$
\begin{aligned}
& 1+G_{M} B_{(s)}=0 \\
& 1+\frac{-5.2551 S+3.5035}{32.75 S^{2}+22.373 S+0.35988} B_{(s)} \\
& 1+\frac{-5.2551 S+3.5035}{32.75 S^{2}+22.373 S+0.35988} K_{C}
\end{aligned}
$$

Desarrollando

$32.75 S^{2}+22.373 S+0.35988+(-5.2551 S+3.5035) \mathrm{K}_{\mathrm{c}}=0$

Ecuación característica

$32.75 \mathrm{~S}^{2}+22.373 \mathrm{~S}+0.35988-5.2551 S \mathrm{~K}_{\mathrm{c}}+3.5035 \mathrm{~K}_{\mathrm{c}}=0$ Nichols

Sintonización por el método de Ziegler-

MARTÍNEZ-MARÍN, Francisco Alejandro, GARCIABADASILVA, Gabriel y HERNÁNDEZ-CERVANTES, Aldo Aarón. Control de presión, modelado matemático y sintonización de controladores por el método de Ziegler-Nichols. Revista de Ingeniería Industrial. 2020 
Sustituyendo iw por S en la ecuación característica

$$
\begin{aligned}
& 32.75(\mathrm{iw})^{2}+22.373(\mathrm{iw})+0.35988-5.2551(i w) \mathrm{K}_{\mathrm{c}} \\
& +3.5035 \mathrm{~K}_{\mathrm{c}}=0 \\
& (-1) 32.75 \mathrm{w}^{2}+\mathrm{i} 22.373 \mathrm{w}+0.35988-i 5.2551 w \mathrm{~K}_{\mathrm{c}} \\
& +3.5035 \mathrm{~K}_{\mathrm{c}}=0 \\
& -32.75 \mathrm{w}^{2}+\mathrm{i} 22.373 \mathrm{w}+0.35988-i 5.2551 w \mathrm{~K}_{\mathrm{c}} \\
& +3.5035 \mathrm{~K}_{\mathrm{c}}=0+0 \mathrm{i}
\end{aligned}
$$

Se iguala la parte imaginaria a cero.

$$
\begin{aligned}
& +\mathrm{i} 22.373 \mathrm{w}-i 5.2551 w \mathrm{~K}_{\mathrm{c}}=0 \mathrm{i} \\
& +22.373 \mathrm{w}-5.2551 w \mathrm{~K}_{\mathrm{c}}=0 \\
& +22.373=5.2551 \mathrm{~K}_{\mathrm{c}} \\
& \mathrm{K}_{\mathrm{u}}=\frac{22.373}{5.2551}=4.257
\end{aligned}
$$

La parte real se iguala a cero.

$$
-32.75 w^{2}+0.35988+3.5035 K_{c}=0
$$

Desarrollando.

$$
\begin{aligned}
& 32.75 \mathrm{w}^{2}=0.35988+3.5035 \mathrm{~K}_{\mathrm{c}} \\
& \mathrm{w}^{2}=\frac{0.35988+3.5035 \mathrm{~K}_{\mathrm{c}}}{32.75}
\end{aligned}
$$

Sustituyendo $K_{c}=4.257$ en (19)

$\mathrm{w}_{\mathrm{u}}=\sqrt{\frac{0.35988+3.5035 \mathrm{~K}_{\mathrm{c}}}{32.75}}=\sqrt{\frac{0.35988+3.5035 * 4.257}{32.75}}=0.682$

$P_{u}=\frac{2 \pi}{\mathrm{w}_{\mathrm{u}}}=\frac{2 \pi}{0.682}=9.212$

\section{Resultados}

Tabla de sintonización para Ziegler-Nichols.

\begin{tabular}{|c|c|c|c|}
\hline & P & PI & PID \\
\hline \hline$K_{C}$ & $\frac{K_{u}}{2}$ & $\frac{K_{u}}{2.2}$ & $\frac{K_{u}}{1.7}$ \\
\hline$\tau_{I}$ & & $\frac{P_{u}}{1.2}$ & $\frac{P_{u}}{2}$ \\
\hline$\tau_{D}$ & & & $\frac{P_{u}}{8}$ \\
\hline
\end{tabular}

Tabla 2

Fuente: Elaboración propia
Para un controlador Proporcional.

$K_{c}=\frac{4.257}{2}=2.128$

Para un controlador Proporcional- Integral.

$K_{c}=\frac{4.257}{2.2}=1.935$

$\tau_{i}=\frac{P_{u}}{1.2}=\frac{9.212}{1.2}=7.676$

Para un controlador ProporcionalIntegral-Derivativo.

$K_{c}=\frac{4.257}{1.7}=2.128$

$\tau_{i}=\frac{P_{u}}{2}=\frac{9.212}{2}=4.606$

$\tau_{D}=\frac{P_{u}}{8}=\frac{9.212}{8}=1.151$

\section{Conclusiones}

En el presente artículo se puede evidenciar que las matemáticas son la herramienta fundamental para el desarrollo de las de las ecuaciones que finalmente nos conducen a los valores del controlador como son la parte proporcional, integral y derivativo.

El método de Ziegler-Nichols en cadena cerrada permite obtener los parámetros de un regulador PID, con resultados muy satisfactorios en la mayoría de los casos en los que es aplicable.

\section{Referencias}

Corripio, S. (1991). Control Automático de Procesos teoría y Prácticas. Editorial Limusa.

Guy, J. L. (1981). Fundamentals of chemicals process dynamics. CE Refresher: Process Dynamics.

Luyben, W. L. (1996). Process Modeling, Simulation, and Control for Chemical Engineers. second Edition.

Ogata, k. (1998). Ingeniería de Control Moderna. Prentice Hall.

MARTÍNEZ-MARÍN, Francisco Alejandro, GARCIABADASILVA, Gabriel y HERNÁNDEZ-CERVANTES, Aldo Aarón. Control de presión, modelado matemático y sintonización de controladores por el método de Ziegler-Nichols. Revista de Ingeniería Industrial. 2020 
Roger G. E. Franks. (1972). Modeling and Simulation in Chemical Engineering. WILEYINTERSCIENCE.

Yunus A. Cengel (2003).Transferencia de Calor.(Segunda Edición).McGraw-Hill 